\title{
ARTICLE
}

\section{In vitro interactions of amphotericin B and non-antifungal com- pounds against opportunistic human pathogen Cryptococcus neoformans}

\author{
Bettina Szerencsés, Anna Mülbacher, Csaba Vágvölgyi, Ilona Pfeiffer* \\ Department of Microbiology, Faculty of Science and Informatics, University of Szeged, Szeged, Hungary
}

\begin{abstract}
The incidence of opportunistic human pathogen Cryptococcus neoformans caused infections have been higher during the last few decades along with the increasing number of susceptible individuals around the world. Recommended treatment of cryptococcal meningoencephalitis is a combined therapy with amphotericin B deoxycholate and flucytosine. Despite of the efficiency of this drug combination, the mortality rate of the disease is high due to the limited accessibility and the high cost of these antifungals in the most severely affected areas. The broad-spectrum activity of non-antifungal drugs and their potential to enhance the efficiency of conventional antifungal agents have been recognised previously. In this study, the in vitro activity of amantadine, valproic acid, trifluoperazine and chlorpromazine was tested against five C. neoformans strains individually and in combination with amphotericin B. All the four compounds exerted slight antifungal activity against the studied C. neoformans strains. Their combination with amphotericin $B$ revealed additive and synergistic interactions. Acta Biol Szeged 63(2):181-184 (2019)
\end{abstract}

\author{
KEY WORDS \\ amphotericin B \\ Cryptococcus neoformans \\ cryptococcosis \\ non-antifungal compounds \\ ARTICLE INFORMATION \\ Submitted \\ 15 October 2019. \\ Accepted \\ 22 November 2019 \\ *Corresponding author \\ E-mail: pfeiffer@bio.u-szeged.hu
}

\section{Introduction}

The encapsulated basidiomycetous yeast, Cryptococcus neoformans is distributed world-wide mainly in association with bird excrement (Srikanta et al. 2014). The species is an opportunistic human pathogen and can cause serious disease primarily in immunocompromised individuals, i.e. HIV-positive patients, patients with organ transplants undergoing immunosuppressive therapy and cancer patients going through chemotherapy; making them vulnerable to fungal infection. The infection of immunocompetent hosts is rare. The disease caused by C. neoformans is called cryptococcosis. The infection starts with the inhalation of the airborne basidiospores or dried cells (Köhler et al. 2015). The spores germinate in the lungs, thereafter the cells disseminated by the blood stream can reach and colonize the central nervous system establishing meningoencephalitis. Cryptococcosis affects about 1 million people in the world - most of them are HIV-infected - and causes the death of more than 600000 patients per year (Warkentien and Crum-Cianfloan 2010). The majority of the cases are registered in certain parts of Africa and Asia where the incidence of HIV-infection is higher (Sloan and Parris 2014).

A combined antifungal therapy involving amphotericin
B deoxycholate and flucytosine is recommended for the treatment of cryptococcal meningoencephalitis (Day et al. 2013). However, flucytosine is an unregistered drug in most parts of Asia and Africa and its cost is high because of the limited number of manufacturers and these factors make the administration of this drug near impossible (Loyse et al. 2013).

Many non-antifungal pharmaceuticals have an antifungal side effect. Some of them can act alone while others can enhance the activity of antifungal agents when used together (Afeltra and Verwe 2003; Judd and Martin 2010; Nyilasi et al. 2010). Among the non-antifungals, the activity of phenothiazines like trifluoperazine and chlorpromazine, have been studied in detail. However, the antifungal activity of amantadine and valproic acid were only recognised recently against opportunistic human pathogenic fungal species (Wood and Nugent 1985; Eilam et al. 1987; Homa et al. 2015; Chaillat et al. 2017). Amantadine is an ion channel blocker used to treat Parkinson's disease (Blanpied et al. 2005). The anti-epileptic drug, valproic acid inhibits the action of histone deacetylases (HDACs) and induces the degradation of HDAC2 (Göttlicher 2004). The antipsychotic drugs chlorpromazine and trifluoperazine exert their antifungal activity via arresting the cell cycle and destroying the cell membrane integrity in the susceptible species (Eilam et al. 1987). All 
Table 1. List of the tested strains

\begin{tabular}{ll}
\hline Species & Strain number \\
\hline Cryptococcus neoformans & IFM 5844 \\
Cryptococcus neoformans & IFO 410 \\
Cryptococcus neoformans & SZMC 26851 \\
Cryptococcus neoformans & SZMC 26852 \\
Cryptococcus neoformans & IFM 48637 \\
\hline
\end{tabular}

IFM: Culture Collection of the Research Centre for Pathogenic Fungi and Microbial Toxicoses, Chiba University, Chiba, Japan

IFO: Institute for Fermentation, Osaka, Japan

SZMC: Szeged Microbiological Collection

these drugs can penetrate across the blood brain barrier and can act in the central nervous system.

The aim of this study was to test the in vitro antiCryptococcus activity of amantadine, chlorpromazine, trifluoperazine and valproic acid against five C. neoformans strains, and to evaluate their interaction with amphotericin B.

\section{Materials and methods}

\section{Yeast strains and growth conditions}

The C. neoformans strains used in the present study are listed in Table 1. The strains were cultivated on Yeast Peptone Dextrose medium (YPD, 0.5\% yeast extract, $1 \%$ peptone, $1 \%$ dextrose, $2 \%$ agar) at $30^{\circ} \mathrm{C}$ for 48 hours and were kept at $4{ }^{\circ} \mathrm{C}$ until use.

The experiments were carried out with actively growing cells; therefore, a single colony was transferred to $2 \mathrm{~mL}$ sterile YPD medium and incubated at $30{ }^{\circ} \mathrm{C}$ for overnight. Cells were then harvested by centrifugation at $10000 \mathrm{rpm}$ for 5 minutes in Heraeus Pico 17 centrifuge (Thermo Scientific, Waltham, MA, US) and washed twice with sterile distilled water, finally they were suspended in RPMI 1640 medium (Sigma-Aldrich, Germany).

\section{Non-antifungal compounds}

Amantadine hydrochloride, chlorpromazine hydrochlo- ride, trifluoperazine hydrochloride, valproic acid sodium salt (Sigma-Aldrich, Germany) and amphotericin B (AppliChem, Darmstadt, Germany) were provided by the manufacturers as standard powder. The non-antifungal compounds were dissolved in $96 \%$ ethanol while amphotericin B in dimethyl sulfoxide (DMSO) to prepare stock solutions $(10 \mathrm{mg} / \mathrm{mL}$ and $1 \mathrm{mg} / \mathrm{mL}$, respectively) which was stored at $-20^{\circ} \mathrm{C}$ until used. Further dilutions were performed in RPMI 1640 medium.

\section{Antifungal activity assays}

\section{Determination of Minimal Inhibitory Concentration (MIC)}

The antifungal effect of the drugs was determined by broth micro-dilution assay in 96-well flat bottom microplate. Fifty $\mu 1$ serially twofold diluted compounds were added to $50 \mu \mathrm{l}$ of standardized cell suspension $\left(8 \times 10^{4} \mathrm{cell} / \mathrm{mL}\right.$ in RPMI 1640 medium). The final concentration of the amphotericin B was ranged from 0.156 to $5.0 \mu \mathrm{g} / \mathrm{mL}$, and those of amantadine, chlorpromazine, trifluoperazine and valproic acid from 7.81 to $500 \mu \mathrm{g} / \mathrm{mL}$. The control samples contained $50 \mu \mathrm{l}$ cell suspension and $50 \mu \mathrm{l}$ RPMI 1640 medium. Solvent control was used to check the effect of the ethanol and DMSO on the growth rate of the strains.

The plates were incubated at $30^{\circ} \mathrm{C}$ for $48 \mathrm{~h}$. At the end of the incubation, the optical density of the samples was detected at $620 \mathrm{~nm}$ in SPECTROstar Nano plate reader (BMG LabTech, Offenburg, Germany). The experiments were carried out at least three times always in triplicates. The MIC was defined as the concentration of the compound caused total inhibition of cell growth.

\section{Interaction between amphotericin $B$ and the non-antifungal compounds}

The in vitro interaction of the compounds and amphotericin B was determined by standard checkerboard titration method. The amphotericin B was tested in a concentration range from 0.156 to $2.5 \mu \mathrm{g} / \mathrm{mL}$ while the concentration ranges of all the other compounds varied from 7.81 to $125 \mu \mathrm{g} / \mathrm{mL}$. The cell concentration in each well was $4 \mathrm{x}$ $10^{4} \mathrm{cell} / \mathrm{mL}$. After the incubation for $48 \mathrm{~h}$ at $30^{\circ} \mathrm{C}$, the optical density of the cultures was detected at $620 \mathrm{~nm}$

Table 2. Antifungal activity of the compounds

\begin{tabular}{|c|c|c|c|c|c|}
\hline \multirow{2}{*}{ Species } & \multicolumn{5}{|c|}{ Minimal inhibitory concentrations $(\mu \mathrm{g} / \mathrm{mL})$} \\
\hline & amphotericin B & amantadine & chlorpromazine & trifluoperazine & valproic acid \\
\hline Cr. neoformans IFM 5844 & 0.625 & $>500$ & 125 & 62.5 & $>500$ \\
\hline Cr. neoformans IFO 410 & 0.625 & $>500$ & 62.5 & 62.5 & $>500$ \\
\hline Cr. neoformans SZMC 26851 & 0.625 & $>500$ & 125 & 62.5 & $>500$ \\
\hline Cr. neoformans SZMC 26852 & 0.625 & $>500$ & 125 & 62.5 & $>500$ \\
\hline Cr. neoformans IFM 48637 & 0.625 & $>500$ & 125 & 62.5 & $>500$ \\
\hline
\end{tabular}


Interactions of amphotericin B and non-antifungal compounds

Table 3. Interaction ratios between amphotericin B and amantadine, chlorpromazine, trifluoperazine and valproic acid after 48 -h incubation at $30^{\circ} \mathrm{C}$

\begin{tabular}{|c|c|c|c|c|c|c|}
\hline & \multirow{2}{*}{ Drugs } & \multicolumn{5}{|c|}{ Strains } \\
\hline & & IFM 5844 & IFO 410 & SZMC 26851 & SZMC 26852 & IFM 48637 \\
\hline \multirow{4}{*}{ AMB + } & amantadine & 0.93 ADD & 1.04 ADD & 0.82 ADD & 0.70 ADD & 0.92 ADD \\
\hline & chlorpromazine & 0.94 ADD & $1.04 \mathrm{ADD}$ & $0.85 \mathrm{ADD}$ & 0.88 ADD & $0.97 \mathrm{ADD}$ \\
\hline & trifluoperazine & 1.29 ADD & $1.02 \mathrm{ADD}$ & $1.51 \mathrm{SYN}$ & 1.29 ADD & 1.09 ADD \\
\hline & valproic acid & $1.07 \mathrm{ADD}$ & 0.86 ADD & $1.88 \mathrm{SYN}$ & 1.12 ADD & $1.55 \mathrm{SYN}$ \\
\hline
\end{tabular}

AMB: amphotericin B; ADD: additive interaction; SYN: synergistic interaction

in SPECTROstar Nano plate reader (BMG LabTech, Offenburg, Germany). The MIC was determined for each compound alone and in combinations. The experiments were carried out at least three times always in triplicates.

\section{Data analysis}

For calculation of the inhibition rates, the absorbencies of the untreated control cultures were assumed to be $100 \%$ growth in each case. Expected efficacy of each combination was determined by the Abbott formula: $\mathrm{I}_{\mathrm{e}}=$ $\mathrm{X}+\mathrm{Y}-(\mathrm{XY} / 100)$, where $\mathrm{I}_{\mathrm{e}}$ is the expected percent inhibition for a given interaction, and $\mathrm{X}$ and $\mathrm{Y}$ are the percent grow th inhibited by the compounds when used alone. The nature of interaction of these antifungal compounds was determined by the interaction ratios (IRs), which were computed as $I R=I_{o} / I_{e}\left(I_{o}\right.$, observed percent inhibition). IRs between 0.5 and 1.5 represent additive interactions, ratios of $>1.5$ represent synergistic interaction, and ratios of $<0.5$ represent antagonistic interactions.

\section{Results}

\section{Antifungal activity of the tested drugs}

The antifungal activities are summarised in Table 2 . All the examined strains were slightly susceptible to the drugs. Among the non-antifungal compounds, the MIC of trifluoperazine proved the lowest: $62.5 \mu \mathrm{g} / \mathrm{mL}$. Chlorpromazine showed the same MIC $(62.5 \mu \mathrm{g} / \mathrm{mL})$ for C. neoformans IFO 410 strain, all the other strains were less susceptible, as the MIC was $125 \mu \mathrm{g} / \mathrm{mL}$ in that case. The MIC of amantadine and valproic acid could not be established as it was out of the applied concentration range. The MIC of amphotericin B was $0.625 \mu \mathrm{g} / \mathrm{mL}$ for each strain.

\section{Interaction between amphotericin B and the non-anti- fungal compounds}

Positive interactions were detected between the amphotericin B and each compound. All the tested drugs augment the effectiveness of the amphotericin B against
C. neoformans strains as additive and synergistic interactions occurred between them (Table 3). Using C. neoformans SZMC 26851 as susceptible strain synergism was detected combining amphotericin $B$ either with valproic acid or trifluoperazine. Valproic acid and amphotericin B combination showed synergistic interaction against IFM 48637 strain too. All the other combinations demonstrated additive interactions between amphotericin B and the drugs against the tested strains.

\section{Discussion}

Cryptococcosis is a world-wide infectious disease associated mainly with immunodeficient hosts. The disease most commonly manifests as cryptococcal meningitis. However, pulmonary and primary cutaneous cryptococcosis also exist (Sloan and Parris 2014). As other invasive fungal infections, cryptococcosis is associated with high morbidity and mortality rate. Particularly the treatment of cryptococcal meningoencephalities affecting the central nervous system is difficult because amphotericin B having significant role in the treatment penetrates poorly across the blood brain barrier due to its relatively high molecular weight (Nau et al. 2010). Additional problem is the low accessibility of the other recommended drug, flucytosine (Loyse et al. 2013).

The in vitro broad-spectrum activity of non-antifungal compounds against human pathogenic fungi was published earlier (Judd and Martin 2009). Testing the activity of phenothiazines such as chlorpromazine and trifluoperazine against medically important yeasts such as C. neoformans proved that it is one of the most susceptible species (Eilam et al. 1987). Although, the anti-Cryptococcus activity of these compounds has been established earlier, their interaction with amphotericin B was not investigated. In this present study, the in vitro action of chlorpromazine, trifluoperazine, valproic acid and amantadine individually and in combination with amphotericin B were studied. The results showed that all the examined compounds possess antifungal activ- 
ity as they slightly reduced the growth of C. neoformans strains when applied alone. Trifluoperazine was the most efficient drug as it had the lowest MIC against all the five strains involved in this study. The drugs and amphotericin B established additive or synergistic interactions as in combination with amphotericin B they achieved more effective growth inhibition than being used alone. Amphotericin B in combination with the studied drugs attained more efficient growth reduction in lower concentrations than used alone.

The positive interaction between the drugs and amphotericin B can be explained by the ability of amphotericin $\mathrm{B}$ to bind to the ergosterol and forming pores in the fungal cell membrane (Gallis et al. 1990). Non-antifungal agents could enter the cells via these pores and could exert their activity within the fungal cell. Amantadine, chlorpromazine, trifluoperazine and valproic acid accumulates in the central nervous system and there is potential to apply them in combination with amphotericin B in the treatment of Cryptococcus-caused meningoencephalitis.

\section{Acknowledgements}

This work was supported by the Hungarian Government and European Union through grant GINOP-2.3.2-15-2016-00012. It is also connected to the project GINOP-2.3.3-15-2016-00006 (Széchenyi 2020 Programme) providing infrastructural development.

\section{References}

Afeltra J, Verwe PE (2003) Antifungal activity of nonantifungal drugs. Eur J Clin Microbiol Infect Dis 22:397-407.

Blanpied TA, Clarke RJ, Johnson JW (2005) Amantadine inhibits NMDA receptors by accelerating channel closure during channel block. J Neurosci 25(13):3312-3322.

Chaillot J, Tebbji F, García C, Wurtele H, Pelletier R, Sellam A (2017) $\mathrm{pH}$-dependant antifungal activity of valproic acid against the human fungal pathogen Candida albicans. Front Microbiol 8:1956. doi: 10.3389/fmicb.2017.01956.

Day JN, Chau TTH, Wolbers M, Mai PP, Dung NT, Mai NH, Phu NH, Nghia HD, Phong ND, Thai CQ, Thai LH, Chuong LV, Sinh DX, Duong VA, Hoang TN, Diep PT, Campbell JI, Sieu TPM, Baker SG, Chau NVV, Hien
TT, Lalloo DG, Farrar JJ (2013) Combination antifungal therapy for cryptococcal meningitis. N Engl J Med 368(14):1291-1302.

Eilam Y, Polacheck I, Ben Gigi G, Chernichovsky D (1987) Activity of phenothiazines against medically important yeasts. Antimicrob Agents Chemother 31(5):834-836.

Gallis HA, Drew RH, Pickard WW (1990) Amphotericin B: 30 years of clinical experience. Rev Infect Dis 2(2):308-329.

Göttlicher M (2004) Valproic acid: an old drug newly discovered as inhibitor of histone deacetylases. Ann Hematol 83(Suppl 1):S91-92.

Homa M, Galgóczy L, Tóth E, Tóth L, Papp T, Chandrasekaran M, Kadaikunnan S, Alharbi NS, Vágvölgyi Cs (2015) In vitro antifungal activity of antipsychotic drugs and their combinations with conventional antifungals against Scedosporium and Pseudallescheria isolates. Med Mycol 53:890-895.

Judd WR, Martin CA (2009) Antifungal activity of nontraditional antifungal agents. Curr Fungal Infect Rep 3(2):86-95.

Kohler JR, Casadevall A, Perfect J (2015) The spectrum of fungi that infects humans. Cold Spring Harb Perspect Med 5:a019273.

Loyse A, Bicanic T, Jarvis JN (2013) Combination antifungal therapy for cryptococcal meningitis. N Engl J Med 368(26):2522.

Nau R, Sörgel F, Eiffert H (2010) Penetration of drugs through the blood-cerebrospinal fluid/blood-brain barrier for treatment of central nervous system infections. Clin Microbiol Rev 23(4):858-883.

Nyilasi I, Kocsubé S, Galgóczy L, Papp T, Pesti M, Vágvölgyi Cs (2010) Effect of different statins on the antifungal activity of polyene antimycotics. Acta Biol Szeged 54(1):33-36.

Sloan D, Parris V (2014) Cryptococcal meningitis: epidemiology and therapeutic options. Clin Epidemiol 6:169-182.

Srikanta D, Santiago-Tirado FH, Doering TL (2014) Cryptococcus neoformans: Historical curiosity to modern pathogen. Yeast 31(2):47-60.

Warkentien T, Crum-Cianflone NF (2010) An update on cryptococcosis among HIV-infected persons. Int J STD AIDS 21(10):679-684.

Wood NC, Nugent KM (1985) Inhibitory effects of chlorpromazine on Candida species. Antimicrob Agents Chemother 27(5):692-694. 